\title{
Influência de alguns fatores sobre o período de gestação no rebanho Girolando da Pesagro (1990-2019)
}

Influence of some factors on the gestation period in the Girolando Pesagro herd (1990-2019)

\section{Osvaldo Almeida Resende ${ }^{1,2^{*}}$; Jaci de Almeida ${ }^{3}$; Rosane Scatamburlo Lizieire Fajardo ${ }^{2}$; Pedro Afonso Moreira Alves'; Sergio Trabali Camargo Filho ${ }^{2}$}

\author{
${ }^{1}$ Embrapa Agrobiologia, RJ; ${ }^{2}$ Pesagro/Cepao, Seropédica, RJ- CEP: 23.890-000, ${ }^{3}$ Centro Universitário de Barra \\ Mansa-UBM, Barra Mansa, RJ
}

\begin{abstract}
Resumo
O rebanho Girolando tem crescido substancialmente nas últimas décadas no Brasil, tornando a raça uma das mais selecionadas e visadas para a exploração de leite entre os criadores. No entanto, é sabido a importância do manejo das fêmeas para evitar perdas durante a gestação, sendo elevado os abortos e as distocias no periparto, por desconhecimento da previsão de partos. Mas para estabelecer uma tabela de previsão de partos é necessário conhecer o período de gestação de raça explorada. Neste contexto, este estudo envolveu observações de três décadas (1990 a 2019) de avaliação dos efeitos de alguns fatores (ano de parto, sexo da cria, grupo sanguíneo materno, raça paterna e ordem de parto) sobre os períodos de gestação (PG) do rebanho Girolando do Centro Estadual de Pesquisa em Agricultura Orgânica (CEPAO), da Empresa de Pesquisa Agropecuária do Estado do Rio de Janeiro (Pesagro-Rio). Para isso, foram utilizados os dados dos registros do rebanho dos sistemas de fichários e software Gera leite ${ }^{\circledR}$, relativos aos PG (dia) de vacas Girolando, segundo as variáveis (1) ano, (2) sexo, (3) grupo sanguíneo materno, (4) raça paterno e (5) ordem de parto, tabulados e submetidos a análises descritivas, a anova one-way e teste Bonferroni no software Bioestat. Os métodos reprodutivos utilizados foram as inseminações, realizadas com a observação do estro, seguindo o sistema tradicional de Trimberg e, na última década, também a inseminação artificial em tempo fixo (IATF), com a utilização de protocolos hormonais para sincronização da ovulação. Os resultados médios, da avaliação de 2.104 partos não mostraram diferenças significativas $(\mathrm{p}>0,05)$, nos efeitos dos fatores analisados sobre os períodos de gestação. Assim, a média geral de $283,2 \pm 6,5$ dias, pode ser considerada como uma estimativa dos partos esperados para o rebanho Girolando da Pesagro, a fim de proporcionar manejo e assistência adequados durante o parto, evitando distocias e perdas de mortalidade no periparto.
\end{abstract}

Palavras chave: Rebanho Girolando, avaliação reprodutiva, período de gestação.

\begin{abstract}
The Girolando herd has grown substantially in recent decades in Brazil, making the breed one of the most selected and targeted for milk exploration among breeders. However, it is known the importance of handling females to avoid losses during pregnancy, with abortions and dystocia in the peri-parturition, being high due to the lack of birth prediction. However, to establish a birth prediction table, it is necessary to know the gestation period of the exploited race. In this context, this study involved observations from three decades (1990 to 2019) to assess the effects of some factors (year of birth, sex of offspring, maternal blood group, paternal race and birth order), on gestation periods (PG) of the Girolando herd of the Centro Estadual de Pesquisa Agricultura Orgânica (CEPAO) of Pesagro-Rio. It is located in the municipality of Seropédica, RJ, in the Baixada Fluminense region, where in general the climate presents high temperatures (maximum $\pm 30^{\circ} \mathrm{C}$ ) and relative air humidity (maximum $<60 \%$ ). For this, data from the herd records of the binder systems and Gera leite ${ }^{\circledR}$ software were used, related to the $P G$ (day) of Girolando cows, according to (1) year, (2) sex, (3) maternal blood group, (4) paternal race and (5) birth order were tabulated and submitted to descriptive analyzes, the one-way anova and Bonferroni test in the Bioestat software. The reproductive methods used were inseminations, performed with the observation of estrus, following the traditional Trimberg system and, in the last decade, also artificial insemination in fixed time (TAI), with the use of hormonal protocols for synchronization of ovulation). The results of the evaluation did not show significant differences ( $p>0.05)$, in the effects of the analyzed factors, on the gestation periods. Thus, the general average of $283.2 \pm 6.5$ days can be considered as an estimate of expected deliveries for the Pesagro Girolando herd, in order to provide
\end{abstract}

Correspondência: osvaldo.resende@embrapa.com.br Recebido: 24 de junho de 2020

Aceito: 14 de junho de 2021 
Resende et al. Influência de alguns fatores sobre o período de gestação no rebanho Girolando da Pesagro (1990-2019).

adequate management and assistance during childbirth, avoid dystocias and mortality losses in the peripartum.

Keywords: Girolando herd, reproductive evaluation, gestation period.

\section{Introdução}

A duração da gestação, o período desde a fertilização efetiva até o parto, é uma característica reprodutiva que afeta significativamente a criação e produção de bovinos. Assim, a avaliação do desempenho zootécnico dos plantéis bovinos são relevantes, para tomadas de decisões na gestão técnico administrativa da exploração da pecuária leiteira. Entretanto, no Brasil, os estudos retrospectivos atualizados, analisando os registros dos bancos de dados são escassos, principalmente em relação aos aspectos reprodutivos na raça Girolando. Como na exploração pecuária leiteira a produtividade é dependente da eficiência reprodutiva dos animais, as observações e registros zootécnicos diários, bem como as análises dos parâmetros reprodutivos devem ser realizadas de forma sistemática e periódicas para avaliar os possíveis desvios e tomar as medidas corretivas (Silva et al., 1992; Norman et al., 2009).

Vários são os parâmetros zootécnicos utilizando índices para avaliação do desempenho reprodutivo (intervalo entre partos, períodos de serviço e de gestação, taxa de concepção, serviço por concepção e idade ao primeiro parto). Entre os mesmos, o período de gestação em comparação com o período de serviço tem tido a sua importância relegada a um plano secundário por ter uma variabilidade pequena dentro das raças. Entretanto, quando se analisa as perdas fetais ao nascimento, verifica-se altas taxas de natimortos e abortos afetando a taxa de natalidade (Carneiro et al., 2010). Este evento evidencia a necessidade de programação de previsão de partos para o rebanho. Para isso é fundamental o conhecimento técnico do período de gestação da raça explorada para o manejo de partos, a fim de prestar assistência aos partos distócicos e evitar perdas de mortalidade no periparto.

$\mathrm{O}$ período de gestação e os fatores que o afetam foram extensivamente investigados em vacas leiteiras, confirmado que diferentes raças têm diferentes períodos de gestação (Silva et al., 1992; Norman et al., 2009). Diversos fatores foram identificados como impactantes no período de gestação, como genética, mês de concepção (ou parto), idade e paridade da matriz, sexo do bezerro, gestações múltiplas (simples versus dupla), número de dias em lactação e nível de produção de leite (Silva et al., 1992; Nogalski e Piwczyński, 2012; Tomasek et al., 2017), confirmando que diferentes raças têm diferentes períodos de gestação. Embora o período de gestação não esteja incluído nas avaliações genéticas na maioria dos países, estudos confirmaram que a variação genética para o período de gestação é grande o suficiente para alterá-la através da seleção (Haile-Mariam e Pryce, 2019). O período de gestação está associado a uma série de características economicamente importantes, incluindo desempenho em lactação, vida produtiva, características de saúde, facilidade de parto e mortalidade de bezerros (Vieira-Neto et al., 2017; Haile-Mariam e Pryce, 2019).

Na literatura há relatos de sete décadas atrás (Alexander, 1950; Brakel et al, 1952; Herman et al., 1953; Foote et al, 1959) reportando a presença dos efeitos de ano, sexo, raça e touro sobre os períodos de gestação. Entretanto, pesquisas realizadas, com a raça Gir na Índia (Lazarus e Anantakrishnan, 1952) e com outras raças (Knapp Junior et al., 1940), indicaram o número de gestações como uma causa sem efeito no período de gestação. No entanto, trabalhos posteriores de autores brasileiros até 1980, só referenciaram à possível influência no sexo dos produtos, não aos demais fatores (Aroeira e Sampaio,1980).

Neste contexto, esse estudo tem o objetivo de avaliar os efeitos de algumas variáveis (ano de parto, sexo da cria, grupo sanguíneo materno, raça paterna e ordem de parto) sobre o período de gestação do rebanho Girolando do Centro Estadual de Pesquisa em Agricultura Orgânica (CEPAO), da Empresa de Pesquisa Agropecuária do Estado do Rio de Janeiro (Pesagro-Rio), durante os anos de 1990 a 2019.

\section{Material e Métodos}

O rebanho bovino da Pesagro-Rio é oriundo do plantel do extinto Instituto de Pesquisa e Experimentação Agropecuária do Centro-Sul (IPEACS) do Ministério da Agricultura, formado por cruzamentos das raças Holandesa, Gir e Guernsey. A empresa está situada em Seropédica, RJ, município da Baixada Fluminense, no $\mathrm{Km} 47$ em Seropédica onde anualmente o clima apresentou-se com elevadas temperaturas (máximas $\pm 30^{\circ} \mathrm{C}$ ) e umidades relativas do ar (máximas $\pm 60 \%$ ). 
Nos últimos quarenta anos optaram-se pelos cruzamentos entre as raças Holandesas $(\mathrm{H})$ e Gir (G) para formar a raça Girolando, para a formação desta raça utilizou-se a inseminação artificial tradicional com o sêmen congelado, o qual ao longo dos anos teve sua qualidade melhoranda ano após ano, impactando diretamente no número de inseminações realizadas em sistema contínuo ou em duas estações anuais.

As inseminações foram realizadas com a observação de cios, seguindo o sistema tradicional de Trimberg (Trimberg e Davis, 1943), e também na última década com o uso de protocolos hormonais. Para isso, as fêmeas ( $>60$ dias de pós-parto e novilhas) da raça Girolando foram sincronizadas em programas de IATF, sendo o protocolo da indução a ovulação realizado em dia aleatório do ciclo estral. Todas as vacas receberam 2,0 mg de Benzoato de Estradiol $\left(\mathrm{BE}^{\mathbb{B}}\right)$ associado ao dispositivo intravaginal de progesterona (monodose) contendo $558 \mathrm{mg}$ de progesterona $\left(\right.$ Cronipres $\left.^{\circledR}\right)$. No D8 os dispositivos foram retirados procedendo-se a administração de $530 \mu \mathrm{g}$ cloprostenol sódico $\left(\right.$ Ciosin $^{\circledR}$ ), mais $1 \mathrm{mg}$ de Cipionato de Estradiol - $\mathrm{ECP}^{\circledR}$ ). As IATFs foram realizadas entre 48-52 horas após a retirada da fonte de progesterona.

Devido aos diversos experimentos realizados, em alguns casos concomitantemente, as estações de reprodução (duas) foram alongadas para poder ter número de animais suficientes à serem trabalhados. A primeira ocorrendo de março a junho e a segunda se setembro a dezembro. Ao final das respectivas estações, as fềmeas não gestantes eram reavaliadas por palpação retal e/ou ultrassonografia e novamente submetidos a IA por mais 2 tentativas. No final de cada ano, as fêmeas não gestantes eram avaliadas para descartes.

O diagnóstico de gestação, nas duas primeiras décadas, era realizado por palpação retal, após os 45 a 60 dias pós inseminação artificial. Já na última década com a massificação dos protocolos hormonais de indução e sincronização de cios e ovulações, somado a necessidade de detectar as fêmeas gestantes o mais precocemente possível, foi utilizado o diagnóstico por ultrassonografia por volta dos 30 a 40 dias, de acordo com os cronogramas dos experimentos. Geralmente eram utilizados os animais que estavam disponíveis, o que nem sempre permitia uma seleção direcionada utilizando todos os parâmetros recomendados para a reprodução. Mesmo se tratando de animais de produção leiteira, não foi avaliada neste estudo a produção dos animais por lactação durante as estações estudadas, sendo este foco de outro estudo posterior. Na primeira estação (março a junho) os animais eram mantidos praticamente à pasto. Já para a segunda estação, devido à redução da quantidade e qualidade das pastagens os animais recebiam suplementação no cocho.

$\mathrm{Na}$ última década o rebanho ativo variou de 396 a 158 animais, sendo estes utilizados com a finalidade de atender as necessidades dos diversos projetos de pesquisas da Empresa. Objetivando estudar as possíveis influências de algumas variáveis sobre os períodos de gestação ocorridos no rebanho Girolando da Pesagro-Rio, no período de 1990 a 2019, foram utilizados os registros nos sistemas de fichários e software Gera leite ${ }^{\circledR}$. Os dados relativos aos períodos de gestações (dias) de vacas Girolando, segundo ano, sexo, grupos sanguíneos materno e paterno e ordem de parto, foram submetidos a análise descritiva, análise de variância e teste de Bonferroni no software Bioestat 5.3 (Ayres et al., 2012).

\section{Resultados e Discussão}

Os resultados médios, desvios e erros padrão para 2.104 períodos de gestação de vacas Girolando (dias), segundo ano, sexo, grupo sanguíneo materno, raça paterna, ordem de parto de 1990 a 2019 são apresentados nas tabelas 1, 2, 3, 4 e 5.

Os dados de registros dos períodos de gestação relativos a abortos $(7,3 \% / 176)$ e natimortos $(5,2 \% / 124)$ não foram incluídos nas avaliações. Entretanto essas taxas estão acima do desejado, sendo necessário tomar as medidas técnicas para redução das mesmas.

As médias de períodos de gestação relativas ao ano de partos (tabela 1) variou de $280,4 \pm 6,2$ a $287,2 \pm 6,7$ dias, não apresentaram diferenças significativas, corroborando com os relatos em outras pesquisas (Leite et al., 1986; Silva et al., 1992), que não evidenciaram efeitos de ano sobre períodos de gestação.

A média geral de $283,2 \pm 6,5$ dias está dentro dos limites de 265 a 295 dias aceitos como período de gestação para animais da raça Girolando (Girolando, 2018), e similar à de 282,4 dias de Silva (1971) para raça Holandesa, e inferiores na Gir as de Villares e Abreu (1948/1949) de 288,9 dias, Aroeira e Sampaio (1980) de 286,8 dias e Leite et al. (1986) de 288,6 dias no Brasil. 
Resende et al. Influência de alguns fatores sobre o período de gestação no rebanho Girolando da Pesagro (1990-2019).

Tabela 1. Médias, desvios padrão e erros padrão do período de gestação de vacas Girolando (PesagroRio), durante os anos de 1990 a 2019.

\begin{tabular}{|c|c|c|c|c|}
\hline $\begin{array}{l}\text { Ano } \\
\text { parto* }\end{array}$ & $\begin{array}{l}\text { Animais } \\
(\mathrm{N})\end{array}$ & $\begin{array}{l}\text { Período gestação* } \\
\text { (Dias) }\end{array}$ & $\begin{array}{l}\text { Desvio padrão } \\
\text { (Dias) }\end{array}$ & $\begin{array}{l}\text { Erro padrão } \\
\text { (Dias) }\end{array}$ \\
\hline 1990 & 15 & 282,5 & 6,7 & 1,7 \\
\hline 1991 & 16 & 284,7 & 7,7 & 1,9 \\
\hline 1992 & 32 & 285,1 & 7,8 & 1,4 \\
\hline 1993 & 47 & 280,8 & 6,4 & 0,9 \\
\hline 1994 & 41 & 280,7 & 7,4 & 1,1 \\
\hline 1995 & 52 & 281,2 & 5,6 & 0,8 \\
\hline 1996 & 72 & 284,5 & 6,9 & 0,8 \\
\hline 1997 & 52 & 284,1 & 7,5 & 1,0 \\
\hline 1998 & 60 & 283,1 & 6,0 & 0,7 \\
\hline 1999 & 92 & 281,7 & 5,4 & 0,6 \\
\hline 2000 & 99 & 282,6 & 7,3 & 0,7 \\
\hline 2001 & 72 & 284,7 & 7,2 & 0,9 \\
\hline 2002 & 91 & 284,1 & 6,5 & 0,7 \\
\hline 2003 & 111 & 282,0 & 6,7 & 0,6 \\
\hline 2004 & 105 & 281,5 & 6,4 & 0,6 \\
\hline 2005 & 89 & 283,5 & 5,6 & 0,7 \\
\hline 2006 & 99 & 282,5 & 7,0 & 0,7 \\
\hline 2007 & 96 & 283,3 & 6,8 & 0,7 \\
\hline 2008 & 83 & 282,3 & 6,7 & 0,7 \\
\hline 2009 & 90 & 283,9 & 5,2 & 0,5 \\
\hline 2010 & 72 & 283,3 & 5,2 & 0,6 \\
\hline 2011 & 116 & 284,2 & 6,1 & 0,6 \\
\hline 2012 & 80 & 280,9 & 5,2 & 0,6 \\
\hline 2013 & 57 & 282,2 & 6,5 & 0,9 \\
\hline 2014 & 76 & 284,7 & 6,7 & 0,8 \\
\hline 2015 & 97 & 280,4 & 6,2 & 0,6 \\
\hline 2016 & 79 & 287,2 & 6,7 & 0,7 \\
\hline 2017 & 47 & 286,2 & 6,5 & 0,9 \\
\hline 2018 & 34 & 285,4 & 5,5 & 0,9 \\
\hline 2019 & 32 & 282,6 & 6,2 & 1,1 \\
\hline Total & 2104 & 283,2 & 6,5 & 1,0 \\
\hline
\end{tabular}

Tabela 2. Médias, desvios padrão e erros padrão dos períodos de gestação de vacas Girolando (PesagroRio), segundo o sexo da cria, durante os anos de 1990 a 2019.

\begin{tabular}{ccccc}
\hline \multirow{2}{*}{ Sexo* } & $\begin{array}{c}\text { Animais } \\
(\mathrm{N})\end{array}$ & $\begin{array}{c}\text { Período gestação** } \\
\text { (Dias) }\end{array}$ & $\begin{array}{c}\text { Desvio padrão } \\
\text { (Dias) }\end{array}$ & $\begin{array}{c}\text { Erro padrão } \\
\text { (Dias) }\end{array}$ \\
\hline Fêmea & 982 & 282,5 & 6,5 & 0,2 \\
Macho & 1122 & 283,5 & 6,6 & 0,2 \\
\hline Total & 2104 & 283,0 & 6,6 & 0,2 \\
\hline
\end{tabular}

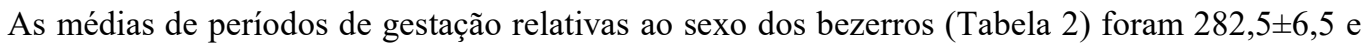
$283,5 \pm 6,5$ dias para fêmeas e machos, respectivamente, não sendo significativa a diferença de um dia. Este resultado é similar aos relatados para a raça Gir, na Índia (Lazarus e Anantakrishnan, 1952) e, no Brasil (Villares e Abreu, 1948/1949; Leite et al., 1986), que não detectaram significâncias nos períodos de partos entre sexos. Resultados contrários foram obtidos por Brakel et al. (1952), Herman et al. (1953), Rollins et al. (1956), Fries et al. (1959), Nadarajah et al. (1989), Silva et al. (1992), que observaram diferenças significativas entre sexos (machos com nascimentos de alguns dias a mais) sobre o período de gestação em outras raças leiteiras.

Em trabalho recente com a raça Holandesa, Atashi e Assaad (2019) verificaram que tanto as categorias primíparas quanto as multíparas gestantes de machos apresentaram maior período de gestação 
Resende et al. Influência de alguns fatores sobre o período de gestação no rebanho Girolando da Pesagro (1990-2019).

que aquelas gestantes de fêmeas $(\mathrm{p}<0,05)$. Sendo a média de período de gestação em vacas primíparas gestantes de machos e fêmeas de $276,68 \pm 0,08$ e $275,71 \pm 0,08$ dias, respectivamente. Já para os valores correspondentes a categoria de multíparas foram $277,58 \pm 0,04$ e 276,59 $\pm 0,03$ para vacas gestantes de machos e fêmeas, respectivamente.

Tabela 3. Médias, desvios padrão e erros padrão dos períodos de gestação de vacas Girolando (PesagroRio), segundo grupo sanguíneo materno, durante os anos de 1990 a 2019.

\begin{tabular}{ccccc}
\hline $\begin{array}{c}\text { Grupo sanguíneo } \\
\text { Materno* }(\% \mathrm{HP})\end{array}$ & $\begin{array}{c}\text { Animais } \\
(\mathrm{N})\end{array}$ & $\begin{array}{c}\text { Período gestação* } \\
\text { (Dias) }\end{array}$ & $\begin{array}{c}\text { Desvio padrão } \\
\text { (Dias) }\end{array}$ & $\begin{array}{c}\text { Erro padrão } \\
\text { (Dias) }\end{array}$ \\
\hline 1 - 01 a 20 & 115 & 282,7 & 6,3 & 0,6 \\
2 - 21 a 40 & 436 & 282,4 & 6,5 & 0,3 \\
3 - 41 a 60 & 442 & 282,3 & 6,6 & 0,3 \\
4 - 61 a 80 & 592 & 283,4 & 7,5 & 0,3 \\
5 - 81 a 100 & 519 & 284,5 & 6,9 & 0,3 \\
\hline Total & 2104 & 283,1 & 6,8 & 0,4 \\
\hline
\end{tabular}

* não significativo teste de Bonferroni - $\mathrm{p}>0,05, \mathrm{~N}=$ = Número; \%: Porcentagem; HP $=$ Holandesa Preta.

As médias de períodos de gestação de vacas Girolando, segundo o grupo sanguíneo materno

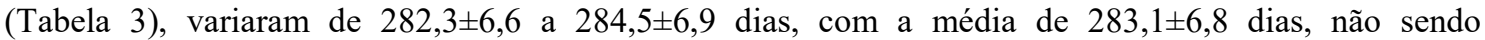
significativas as diferenças, para maior ou menor de grau participação das raças Holandesa e Gir leiteira nos grupos sanguíneos avaliados. Estes resultados são ligeiramente inferiores ao da raça Holandesa (Silva, 1971) e Gir (Villares e Abreu, 1948/1949; Aroeira e Sampaio, 1980; Leite et al., 1986), porém dentro dos padrões da raça Girolando, para registros de partos (Girolando, 2018).

Os valores de estimativas de herdabilidade para o fator materno, face a baixa repetibilidade, indicam que o efeito da vaca não é predominante na variabilidade genética do período de gestação (Norman et al., 2009, Haile-Mariam e Pryce, 2019).

Tabela 4. Médias, desvios padrão e erros padrão dos períodos de gestação de vacas Girolando (PesagroRio), segundo a raça paterna, durante os anos de 1990 a 2019.

\begin{tabular}{lcccc}
\hline Raça Paterna* & $\begin{array}{c}\text { Animais } \\
(\mathrm{N})\end{array}$ & $\begin{array}{c}\text { Período gestação* } \\
\text { (Dias) }\end{array}$ & $\begin{array}{c}\text { Desvio padrão } \\
\text { (Dias) }\end{array}$ & $\begin{array}{c}\text { Erro padrão } \\
\text { (Dias) }\end{array}$ \\
\hline Holandesa (HP) & 1118 & 282,2 & 6,1 & 0,2 \\
GO (5/8HP) & 65 & 284,4 & 7,1 & 0,9 \\
Gir leiteiro & 596 & 285,3 & 6,9 & 0,3 \\
Ignorada & 325 & 282,7 & 8,9 & 0,5 \\
\hline Total & 2104 & 283,7 & 7,3 & 0,5 \\
\hline
\end{tabular}

* não significativo teste de Bonferroni - $\mathrm{p}>0,05 ; \mathrm{N}=$ Número; $\mathrm{HP}=$ Holandesa Preta e $\mathrm{GO}=$ Girolando.

As médias de períodos de gestação de vacas Girolando segundo raça paterna (Tabela 4) variaram de $282,2 \pm 6,1$ dias para a raça Holandesa a $285,3 \pm 6,9$ dias para a raça Gir Leiteiro, com a média de $283,7 \pm 7,3$ dias, não havendo diferenças significativas entre médias das raças dos touros utilizados nos cruzamentos. Apesar de haver extensa literatura (Alexander, 1950; Brakel et al.,1952; Foote et al., 1959; Norman et al., 2011) relatando que existe diferença e/ou influência do touro sobre o período de gestação das vacas, em alguns dos trabalhos com gado indiano, tal efeito não foi detectado (Briquet Júnior e Abreu, 1948; Villares e Abreu, 1948/1949), corroborando plenamente, como resultado deste estudo.

Trabalhos mais recentes com a raça Holandesa encontraram períodos de gestação menores, 281,3 dias (Nadarajah et al., 1989), 280 dias (Silva et al., 1992), 281,6 dias (Norman et al., 2009), 279,1 dias (Nogalski e Piwczyński, 2012) e 278,1 dias (Atashi e Assaad, 2019).

De acordo com Nogalski e Piwczyński (2012) a duração da gestação de vacas Guernsey, Holandesas e Jersey em várias fazendas da Flórida aumentou em quatro dias, em média, durante um período de 50 anos. Segundo os autores Silva et al. (1992), esse aumento pode ser atribuído a um acréscimo acentuado nos níveis de produção. A gestação mais curta foi observada nos meses de verão, e esses achados com os resultados de pesquisas anteriores, validaram o efeito de temperaturas mais altas.

Em pesquisa realizada pela Livestock Improvement Corporation na Nova Zelândia (Lic., 2016) verificou-se que ao avaliar os dados do programa de prova de touros, alguns reprodutores têm valor genético para período de gestação de -9 dias; isto é, eles podem reduzir o período de gestação em até 9 
Resende et al. Influência de alguns fatores sobre o período de gestação no rebanho Girolando da Pesagro (1990-2019).

dias, o que favorece o recrescimento de vacas em sistemas de produção sazonal, enquanto outros têm um valor de reprodução para a duração da gestação de +5 dias.

Sabe-se atualmente, que a diferença de períodos de gestação para as raças é devido às habilidades de transmissão prevista (PTA-Predicted Transmitting Abilities) dos touros utilizados no rebanho. As PTAs para a duração da gestação (GL-Gestation Length) foram desenvolvidas para todas as raças leiteiras e mestiços (Wright e Van Raden, 2017). Segundo Wright e Van Raden (2017) as PTAs e GL para touros da raça Holandesa (nascidos em 1995 ou mais tarde com 90\% ou mais de confiabilidade) têm um mínimo de $-5,6$ e um máximo de $+6,4$ e um desvio padrão de cerca de 1,4 dias. O GL curto está correlacionado favoravelmente em cerca de $0,38 \mathrm{com}$ a facilidade de parto da filha e em cerca de 0,24 a 0,29 com rendimento e vida produtiva, sendo que, a forte seleção atual para essas características correlatas diminuiu o GL nos últimos anos (Wright e Van Raden, 2017). Os autores relataram ainda que a duração da gestação (GL) pode ser útil nos programas de acasalamento para agrupar todas as datas de nascimento em partos sazonais, administrar maternidades ou melhorar a facilidade do parto, como uma característica correlacionada.

Assim, existem aplicativos de gerenciamento em potencial para conhecer a PTA da duração da gestação de um pai, mas a duração média da gestação é uma característica que deve considerar mudar ao longo do tempo. Provavelmente não para a maioria das raças, pois a maioria das pesquisas sugere que a duração da gestação é uma característica com um ótimo intermediário (Dechow, 2017). Ainda segundo o autor, uma duração de gestação muito longa aumenta a probabilidade de dificuldade de parto. Da mesma forma, períodos de gestação ligeiramente menores, que a média estão associados a pontuações de dificuldade para parto um pouco melhoradas, mas se longe demais em direção à curta duração da gestação, pode-se observar um aumento nas taxas de natimortos (Dechow, 2017). Os períodos de gestação abaixo da média atual, também estão associados a menor produção de leite, gordura e proteína, do que a média um pouco acima da média do tempo de gestação nas Holandesas, e no geral, parece que não existem benefícios da seleção para uma duração de gestação diferente das médias (Dechow, 2017).

Tabela 5. Médias, desvios padrão e erro padrão do período de gestação de vacas Girolando (Pesagro-Rio), segundo ordem de parto, durante os anos de 1990 a 2019.

\begin{tabular}{ccccc}
\hline Ordem de Parto* & $\begin{array}{c}\text { Animais } \\
(\mathrm{N})\end{array}$ & $\begin{array}{c}\text { Período gestação* } \\
\text { (Dias) }\end{array}$ & $\begin{array}{c}\text { Desvio padrão } \\
\text { (Dias) }\end{array}$ & $\begin{array}{c}\text { Erro padrão } \\
\text { (Dias) }\end{array}$ \\
\hline $1^{\underline{\underline{o}}}$ & 602 & 282,5 & 6,4 & 0,3 \\
$2^{\mathrm{o}}$ & 505 & 283,1 & 6,6 & 0,3 \\
$3^{\mathrm{o}}$ & 378 & 282,8 & 6,5 & 0,3 \\
$4^{\mathrm{o}}$ & 265 & 283,9 & 6,8 & 0,4 \\
$5^{\mathrm{o}}$ & 178 & 284,0 & 6,5 & 0,5 \\
$6^{\mathrm{o}}$ & 103 & 284,8 & 7,0 & 0,7 \\
$7^{\mathrm{o}}$ & 46 & 282,9 & 7,0 & 1,1 \\
$8^{\underline{o}}$ & 19 & 283,0 & 4,9 & 2,4 \\
\hline $9^{-}$ & 8 & 282,8 & 6,8 & 0,8 \\
\hline
\end{tabular}

* não significativo teste de Bonferroni - $\mathrm{p}>0,05, \mathrm{~N}=$ Número.

Segundo a ordem de parto as médias de período de gestação de vacas Girolando (Tabela 5) variaram de $282,5 \pm 6,4$ a $284,8 \pm 7,0$ dias, com a média de 283,3 $\pm 6,5$ dias, não sendo significativas as diferenças, encontradas do primeiro ao nono parto avaliados. Médias diferentes em raças leiteiras foram obtidos por Knapp Junior et al. (1940); Lazarus e Anantakrishnan (1952), na raça Sindi (Singh e Ray, 1961) e na raça Gir (Leite et al., 1986), estes autores também não encontraram significâncias entre as médias na ordem de partos. Entretanto, trabalhos realizados por Herman et al. (1953) Rollins et al. (1956), e Foote et al. (1959), relataram diferenças significâncias entre as médias de períodos de gestação para ordem de parto.

Já Andersen e Plum (1965), em uma revisão sobre período de gestação e peso ao nascimento concluíram que, o período da gestação e o peso ao nascer foram influenciados pela hereditariedade. A duração da gestação é mais herdável que o peso ao nascer, indicando que tanto a duração da gestação, quanto o peso ao nascer são mais influenciados pelo bezerro do que pela mãe. Por esse motivo, a seleção entre os bezerros a favor ou contra curto período da gestação e o pequeno peso ao nascer devem produzir mudanças razoavelmente rápidas. 
Resende et al. Influência de alguns fatores sobre o período de gestação no rebanho Girolando da Pesagro (1990-2019).

Neste trabalho não foram observadas diferenças significativas dos fatores estudados sobre os períodos de gestação, provavelmente pelo manejo à pasto e produtividade média das vacas (em torno de \pm $3000 \mathrm{~kg} /$ lactação) não avaliadas. No entanto, a alta produção de leite prolonga a gestação, como sugerido por uma correlação genética positiva entre período de gestação e níveis de produção de leite (Silva et al., 1992), e altas temperaturas no verão aceleram o parto e diminuem a duração da gestação (McClintock et al., 2003).

\section{Conclusões}

$\mathrm{Na}$ avaliação não foram observadas diferenças significativas nos períodos de gestação dos parâmetros ano, sexo, grupos sanguíneos maternos, raça paterno e ordem de parto. O período médio da gestação de geral de $283,2 \pm 6,5$ dias pode ser considerado como estimativa de previsão de partos para o rebanho Girolando do CEPAO da Pesagro-Rio, a fim de prestar manejo e assistência adequados aos partos, evitando distocias e perdas por mortalidade no periparto.

\section{Referências}

Alexander MH. Length of gestation in the five major breeds of dairy cattle. J Dairy Sci, v.33, n.6, p. 377-378, 1950.

Andersen H., Plum M. Gestation length and birth weight in cattle and buffaloes. J Dairy Sci, Champaign, v.48, n.9, p.1224-1235, 1965.

Aroeira JADC, Sampaio IBM. Duração da gestação em gado zebu. Pesq Agropec Bras, Brasília, v.1, n.2, p.167-169, 1980.

Atashi H, Asaadi A. Association between gestation length and lactation performance, lactation curve, calf birth weight and dystocia in Holstein dairy cows in Iran. Anim Reprod, v.16, n.4, p.846-852, 2019. https://doi.org/10.21451/1984-3143-ar2019-0005

Ayres M, Ayres Jr M, Ayres DL, Santos AAS. BioEstat 5.0. Aplicações estatísticas nas áreas das ciências bio-médicas, 2012 Acessivel em: mamiraua.gov.br.

Brakel WJ, Rife DC, Salisbury SM. Factors associated with the duration of gestation in dairy cattle. J Dairy Sci, v.35, n.3, p.179-194, 1952.

Briquet Junior, Abreu J. Sobre o período de gestação nas vacas zebuínas. 1. Raça Guzerá. Rer. Agric, Piracicaba, v.23, n.7-8, p.219-236, 1948.

Carneiro MA, Bergamaschi M, Machado R, Barbosa RT. Eficiência reprodutiva das vacas leiteiras. Circular técnica 64, EMBRAPA, São Carlos, SP, 12 p, 2010.

Dechow C. Gestation length evaluations are now available. Hoard's Dairyman. Oct. 25. 2017. https://hoards.com/print-article-22003-permanent.htm. Acesso em: 20.06.2020

Foote WD, Tyler WJ, Casida LE. Effect of some genetic and maternal environmental variations on birth weight and gestation length in Holstein cattle. J Dairy Sci, v.42, n. 2, p.305-311, 1959.

Fries JC, Touchberry RW, Hays RL. Heritability of length of the gestation period in dairy cattle. J Dairy Sci, v.42, n.4, p.598-606, 1959.

Girolando. Girolando Serviço de Registro Genealógico da Raça Girolando - SRGRG, p.18, 2018.

Haile-Mariam M, Pryce J. Genetic evaluation of gestation length and its use in managing calving patterns. J Dairy Sci, v.102, n.1, p.476-487, 2019. http://dx.doi.org/10.3168/jds.2018-14981

Herman HA, Spalding RW, Bower KW. Factors affecting length of gestation period in dairy cattle touchberry. Missouri. Agr. Expt. Sta, Res. Bull, n.529, 14p., 1953.

Knapp Junior B, Lambert WN, Black NH. Factors influencing length of gestation and birth weight in cattle. J Agric Res, v.61, n.4, p.277-285, 1940.

Lazarus AJ, Anantakrishnan CP. Observations on some Indian cattle. Part 1. The period of gestation in cows. Indian J Dairy Sci, Madras, v.24, n.1, p.5-24, 1952.

Leite PRM, Bellido MM, Paca FR, Santos ES. Fatores que influenciam o período de gestação e intervalo entre partos de vacas Gir no nordeste brasileiro. Pesq Agropec Bras, Brasília, v.2, n.1, p.87-92, 1986.

LIC - Livestock Improvement Corporation. Short gestation duration. Livestock Breeding Corporation. Available at: http://www.lic.co.nz/lic_Premier_Sires_Teams.cfm (2016). Acessado em maio de 2020.

McClintock B, Gilmour K, Goddārd A. Relationships between calving traits in heifers and mature cows in Australia. Proceedings of the 2003 Interbull meeting, n.31, p.102-106, 2003. 
Resende et al. Influência de alguns fatores sobre o período de gestação no rebanho Girolando da Pesagro (1990-2019).

Nadarajah K, Burnside EB, Schaeffer LR. Factors affecting pregnancy duration in Ontario Holsteins. Can. J Anim Sci, v.69, n.4 p.1083-1086, 1989. http://dx.doi.org/10.4141/cjas89-123

Nogalski Z, Piwczyński, D. Association of pregnancy time with other reproductive characteristics in dairy cattle. Asiático-Australas. J Anim Sci, Jan, v.25, n.1, p.22-27, 2012.

http://dx.doi.org/10.5713/ajas.2011.11084

Norman HD, Wright JR, Kuhn MT, Hubbard SM, Cole JB, Van Raden PM. Genetic and environmental factors that affect the duration of pregnancy in dairy cattle. J Dairy Sci, v.92, v.5, p.22592269, 2009. http://dx.doi.org/10.3168/jds.2007-0982.

Norman HD, Wright JR, Miller RH. Potential consequences of selection to change gestation length on performance of Holstein cows. J Dairy Sci, v.94, n.2, Feb, p.1005-1010, 2011. http://dx.doi.org/10.3168/jds.2010-3732

Rollins WC, Laben RC, Mear SW. Gestation length in inbred Jersey herd. J Dairy Sci, v.39, n.11, p.1578-1593, 1956.

Silva RG. Estudos sobre a duração do período de gestação nas raças Jersey e Holandesa PB no estado do Rio de Janeiro. Pesq Agropec Bras, v.6, n.1, p.79-85, 1971.

Silva HMCJ, Wilcox CJ, Thatcher WW, Becker RB, Morse D. Factors affecting days open, gestation length, and calving interval in Florida dairy cattle. J Dairy Sci, v.75, n.1, p.288-293, 1992. http://dx.doi.org/10.3168/jds.S0022-0302(92)77764-9

Singh RS, Ray SM. Studies on the gestation period of dairy cows. The variability of the length of gestation in Red Sindhi cows. Indian J Dairy Sci, v.14, n.1, 1961.

Trimberg W, Davis H.P. Conception rate in dairy cattle by artificial insemination at various stages of estrus. Research Bulletin of the University of Nebraska, Lincoln. n.129, 14 p., 1943.

Tomasek R, Rezac P, Havlicek Z. Environmental and animal factors associated with gestation length in Holstein cows and heifers in two herds in the Czech Republic. Theriogenology, v.87, v.1, p.100-107, 2017. http://dx.doi.org/10.1016/j.theriogenology.2016.08.009

Vieira-Neto A, Galvão K, Thatcher W, Santos J. Association among gestation length and health, production, and reproduction in Holstein cows and implications for their offspring. J Dairy Sci, v.100, n.4, p.3166-3181, 2017. http://dx.doi.org/10.3168/jds.2016-11867

Villares JB, Abreu J. Contribuição para o estudo do período de gestação nas raças Gir e Indubrasil. Bol Industr Anim, São Paulo, v.10, n.1, p.30-40, 1948/1949.

Wright JR, Van Raden PM. Genetic evaluation of gestation length as a trait of the service sire. J Dairy Sci, v. 100 (Suppl. 2), n. 42 (abstr. M100), 2017.

https://queries.uscdcb.com/News/gestation_length_07_06_2017.htm Acesso: 20.06.2020 